\title{
SUPPORTING INFORMATION Molecular Mechanism of Cell Membrane Protection by Sugars: A Study of Interfacial H-bond Networks
}

Xiao You, ${ }^{1}$ Euihyun Lee, ${ }^{1}$ Cong Xu, ${ }^{2}$ Carlos R. Baiz ${ }^{1 *}$

${ }^{1}$ Department of Chemistry, the University of Texas at Austin, Austin, TX, USA

${ }^{1}$ Department of Physics, the University of Texas at Austin, Austin, TX, USA

\section{S1. Sample Preparation}

The lipid 1,2-dimyristoyl-sn-glycero-3-phosphocholine (DMPC) $25 \mathrm{mg} / \mathrm{mL}$ chloroform was purchased from Avanti Polar Lipids (Birmingham, AL), stored at $-25^{\circ} \mathrm{C}$, and used without further purification. The chloroform solvent was fully evaporated before use by passing a dry nitrogen stream followed by desiccation under a mild vacuum. DMPC lipids were then reconstituted in glucose in $\mathrm{D}_{2} \mathrm{O}$ solutions with varying glucose concentrations from 0 to $600 \mathrm{mg} / \mathrm{mL}$. This procedure ensures that the sugar concentration is the same inside and outside the vesicle. The lipid concentration was kept at $50 \mathrm{mg} / \mathrm{mL}$ for all solutions. Reconstituted samples were subject to six freeze-thaw cycles, 20-minute sonication, and extrusion through $100-\mathrm{nm}$-pore filters at $60^{\circ} \mathrm{C}$ to obtain uniform $100-\mathrm{nm}$ vesicles.

Ethyl Acetate (EtOAc) solutions $\mathrm{D}_{2} \mathrm{O}$ solutions were prepared at the same glucose concentration as the DMPC lipids with an EtOAc concentration of $15 \mathrm{mg} / \mathrm{mL}$.

\section{S2. Infrared Absorption Spectroscopy}

\section{Fourier-transform infrared spectroscopy (FTIR)}

FTIR spectra were recorded on a Bruker Vertex 70 spectrometer. The spectrometer and sample chamber were purged with dry air to minimize perturbations from water vapor. Samples were held between two CaF2 windows separated by a $25 \mu \mathrm{m}$ PTFE spacer in a brass sample cell. The sample temperature-controlled sample cell was equilibrated for 5 minutes at each temperature setpoint, using a recirculating chiller. Spectra were measured 
at following temperatures: $5,10,15,20,21,22,23,24,25,26,27,28,29,30,35,40,45$, $50,55,60,65,70,80^{\circ} \mathrm{C}$. Sample FTIR spectra are shown in the main text (Figure 1B).

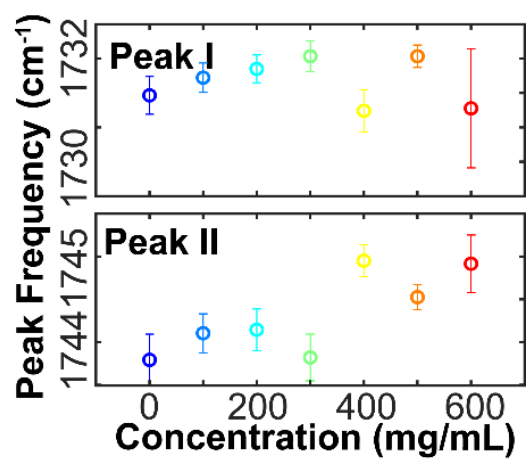

Figure S1. DMPC ester carbonyl peak positions of vesicles across the sugar concentration range extracted from two Gaussian fits of the absorption spectra as shown in the main text Figure 1B. Peak I and Peak II correspond to carbonyls with 1 and 0 HB respectively. B) Average number of H-bond each carbonyl group form in DMPC and EtOAc solutions across the sugar concentration range extracted by the oscillator-strength-weighted $\left(\frac{\varepsilon_{1 H B}}{\varepsilon_{0 H B}}=\right.$ 1.49) areas of the two peaks as described previously. ${ }^{1}$


Figure S2. A) Normalized FTIR spectrum of ethyl acetate in the ester carbonyl stretching frequency region shown as an example. The ester carbonyl peak in $\mathrm{D}_{2} \mathrm{O}$ is well described by a sum of two Gaussians (shaded peaks) representing the 2HB and 1HB carbonyl ensembles as labeled in the figure. (B) Center frequencies of the Gaussians peaks across the glucose concentration range. 


\section{S3. Temperature Dependent FTIR}

\section{Determination of $\mathbf{H}$-bond Enthalpies}

The interfacial H-bond network can be characterized through IR spectroscopy of the lipid vibrational modes. Temperature-dependent IR absorption provides direct access to the $\mathrm{H}$ bond formation thermodynamics. ${ }^{2-3}$ The areas of the two individual peaks composing the ester carbonyl band corresponds to the H-bonds populations when weighted by the oscillator-strengths of the carbonyl in the 0 and $1 \mathrm{H}$-bond configuration (Figure 1A, main text) ${ }^{4}$

The interconversion between the two H-bond species can be represented as:

$$
1 \mathrm{HB} \rightleftharpoons 0 \mathrm{HB}+\mathrm{D}_{2} \mathrm{O}
$$

Equation 1

The equilibrium constant of this process is thus given by

$$
K=\frac{a_{0 H B} \cdot a_{D_{2} O}}{a_{1 H B}}
$$

Equation 2

where $a$ is the activity of each of the components as shown in Eq.1. As the concentration of carbonyl groups is very low $(<0.25 \mathrm{M})$, the activity coefficients can be assumed to be unitary for all the species in solution, and the water concentration is constant. Thus, Eq. 2 can be simplified to

$$
K^{\prime}=\frac{[0 H B]_{e q}}{[1 H B]_{e q}}=\frac{k_{1}}{k_{2}}
$$

Equation 3

where $K^{\prime}$ is $K /\left[\mathrm{D}_{2} \mathrm{O}\right]$ and $\mathrm{k}_{1}$ and $\mathrm{k}_{2}$ represent the forward and reverse rate constants for Equation 1 above.

The Gibbs free energy of any chemical process, including the formation of H-bond, is defined as

$$
\Delta G^{0}=\Delta H^{0}-T \Delta S^{0}
$$

Equation 4

where $\Delta H^{0}$ and $\Delta S^{0}$ represent the changes in the enthalpy and entropy in standard conditions, respectively. $T$ is the temperature in the Kelvin unit. The Gibbs free energy $\Delta G^{0}$, under equilibrium conditions, can be expressed as: 


$$
\Delta G^{0}=-R T \ln \left(K^{\prime}\right)
$$

Equation 5

where $R$ is the gas constant, $T$ is the temperature, and $K^{\prime}$ is the equilibrium constant of the process. Combining the Eq. 3 - 5 above, the relationship between the equilibrium constant and the changes in the enthalpy and entropy of the process is found

$$
\ln \left(K^{\prime}\right)=\ln \left(\frac{[0 H B]}{[1 H B]}\right)=-\frac{\Delta H^{0}}{R T}+\frac{\Delta S}{R}
$$

Equation 6

According to the Lambert-Beer law, the IR absorption $A_{i}$ of a sample is determined by the molar absorptivity coefficient $\varepsilon_{i}$, the concentration of the species $[i]$, and the sample path length $b$ :

$$
A_{i}=\varepsilon_{i} b[i]
$$

Equation 7

Combine the Eq. 6 and Eq.7 above, the relationship between the absorption of the species and the thermodynamics of the chemical process

$$
\ln \left(\frac{A_{0 H B}}{A_{1 H B}}\right)=-\frac{\Delta H^{0}}{R T}+\frac{\Delta S}{R}-\ln \left(\frac{\varepsilon_{0 H B}}{\varepsilon_{1 H B}}\right)=-\frac{\Delta H^{0}}{R} \cdot \frac{1}{T}+\left[\frac{\Delta S}{R}-\ln \left(\frac{\varepsilon_{0 H B}}{\varepsilon_{1 H B}}\right)\right] \quad \text { Equation } 8
$$

As we can see, Eq. 8 can be written as the form of $y=a x+b$, where $\mathrm{y}$ is $\ln \left(\frac{A_{0 H B}}{A_{1 H B}}\right), \mathrm{x}$ is $\frac{1}{T}$, a is the $-\frac{\Delta H^{0}}{R}$, and $\mathrm{b}$ is the intercept $\left[\frac{\Delta S}{R}-\ln \left(\frac{\varepsilon_{0 H B}}{\varepsilon_{1 H B}}\right)\right]$.

Here, we plot $\ln \left(\frac{A_{0 H B}}{A_{1 H B}}\right)$ against $\frac{1}{T}$. The experimental data is then fitted via linear regression. 

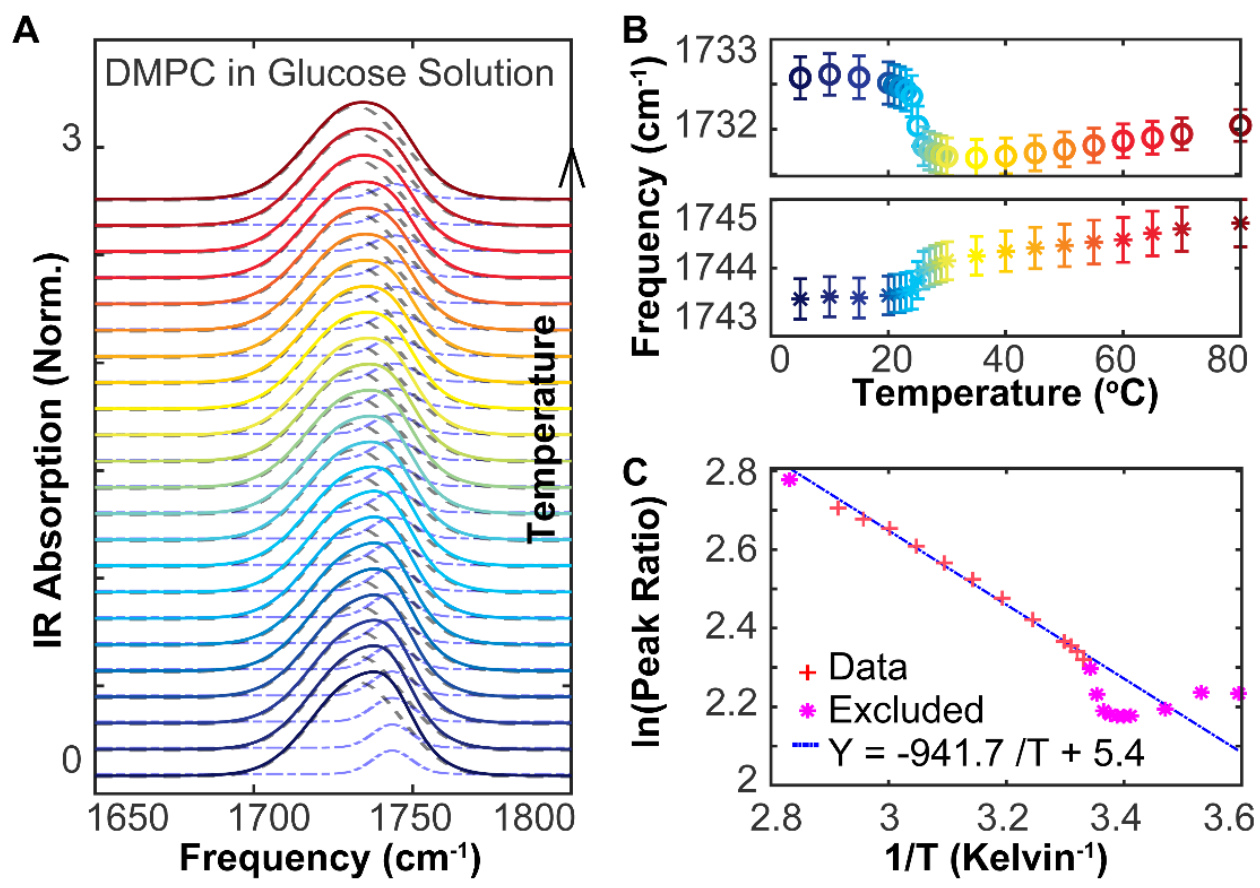

Figure S3. A) Temperature-dependent FTIR spectra with two-Gaussian fit. The color of each spectrum corresponds to the temperatures shown in B. B) Gaussian peak centers at each temperature show liquid-to-gel phase transition of lipids; C) Van't Hoff plots of DMPC carbonyl vibrations show a linear dependence of $\ln$ (peak ratio) to $1 /$ temperature with linear fitting. The slope reports the H-bond enthalpy (Equation 8)
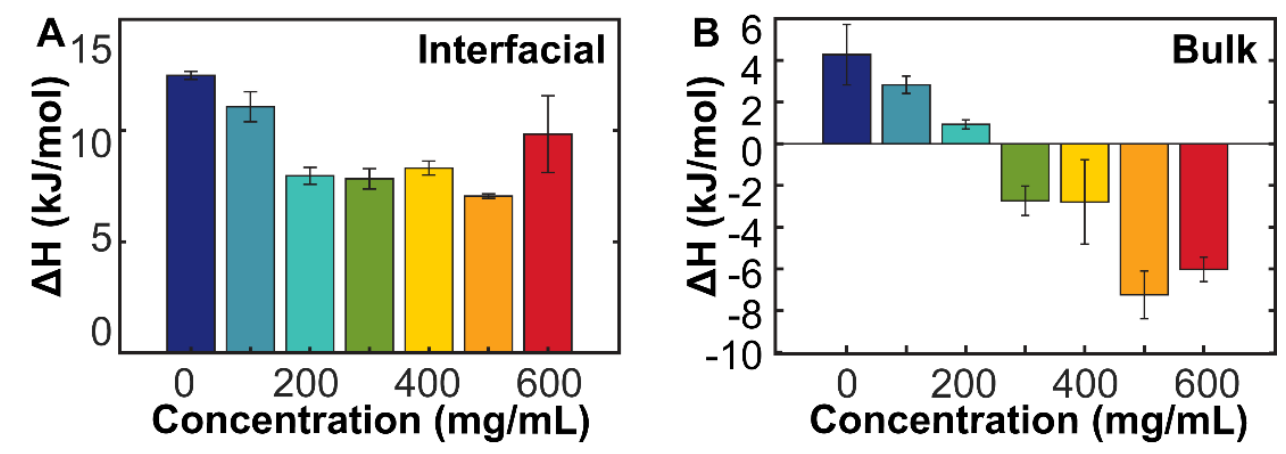

Figure S4. Enthalpy of A) DMPC in the glucose-water solutions. B) Ethyl acetate in glucose-water solutions. 


\section{S4. Ultrafast 2D IR Spectroscopy}

Ultrafast 2D IR spectra were measured using a pulse-shaper-based 2D IR spectrometer described previously. ${ }^{5}$ In brief, 2D IR measurements consist of excitation pulses (pump) and a detection pulse (probe) resonant with the carbonyl stretching mode. 2D IR spectra show the characteristic positive-negative peak doublet that arises from the ground-state bleach (red contours, diagonal) and excited-state absorption contributions (blue contours, below-diagonal) as shown in Figure 2A (main text). Initially the peaks are diagonallyelongated indicating that the pump and probe frequencies are correlated. This correlation between the pump and probe decreases over time as a result of spectral diffusion of the carbonyl. Nodal-line slope (NLS) analysis is used to quantify the diagonal elongation. ${ }^{6}$ The time evolution of the NLS as a function of waiting time, $\mathrm{t}_{2}$ (Figure $2 \mathrm{~B}$, main text), represents the frequency-frequency correlation function of the carbonyls.

Pump and probe pulses were centered at $1700 \mathrm{~cm}^{-1}$ with an FWHM of $300 \mathrm{~cm}^{-1}$. Coherence times $\left(t_{1}\right)$ were scanned up to $3 \mathrm{ps}$ in 20 fs steps. Spectra were measured at a series of waiting times, $\mathrm{t}_{2}$, from 50 fs to 5000 fs. The pump and probe pulses were maintained at perpendicular polarizations to reduce pump scatter. Experimental temperature was set to $60{ }^{\circ} \mathrm{C}$ to maintain the DMPC lipid well above its phase transition temperature.

The NLS was calculated by mapping the probe frequency, $\omega_{3}$, at each pump frequency, $\omega_{1}$, corresponding to the node between the positive and negative peaks. The resulting scatter plots are fit to a line, and the slope is extracted from each fit.

A

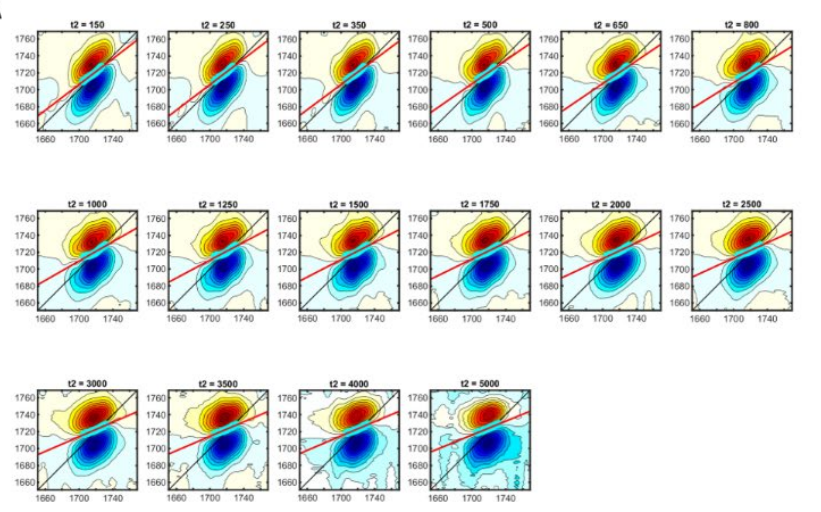

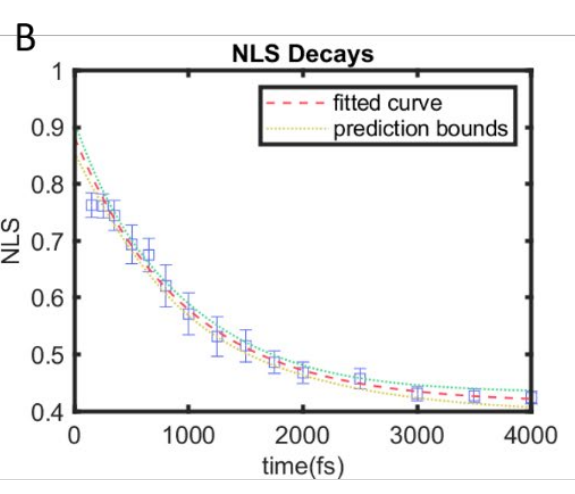


Figure S5. A) 2D IR spectra of the DMPC carbonyl stretch in $\mathrm{D}_{2} \mathrm{O}$ at selected waiting time delays $\left(t_{2}\right)$. The horizontal axis represents the pump frequency $\left(\omega_{1}\right)$, and the vertical axis is the probe frequency $\left(\omega_{3}\right)$. B) Nodal line slope analysis of the corresponding 2D IR spectra. The red solid line represents a monoexponential fit with an offset to the waiting-timedependent NLS.

A
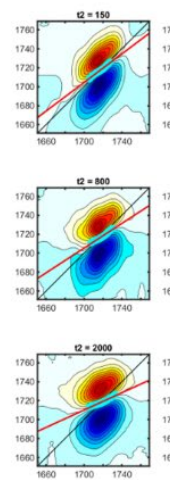
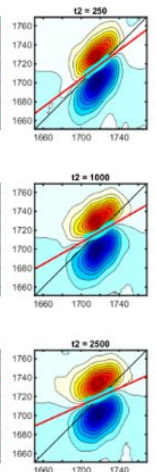
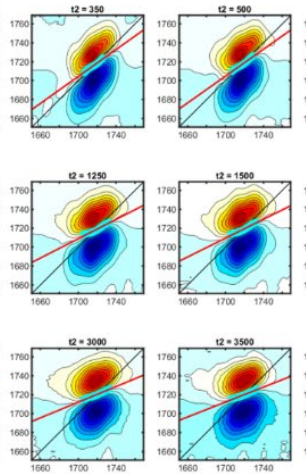


Figure S6. A) 2D IR spectra of the DMPC carbonyl stretch in $100 \mathrm{mg} / \mathrm{mL}$ glucose solutions in $\mathrm{D}_{2} \mathrm{O}$ at selected waiting time delays $\left(\mathrm{t}_{2}\right)$. The horizontal axis represents the pump frequency $\left(\omega_{1}\right)$, and the vertical axis is the probe frequency $\left(\omega_{3}\right)$. B) Nodal line slope analysis of the corresponding 2D IR spectra. The red solid line represents a monoexponential fit with an offset to the waiting-time-dependent NLS.

A
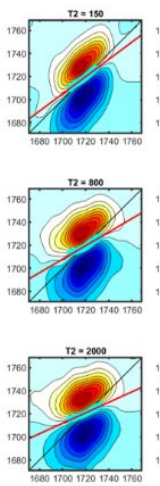
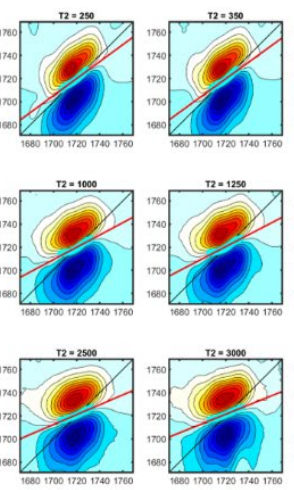
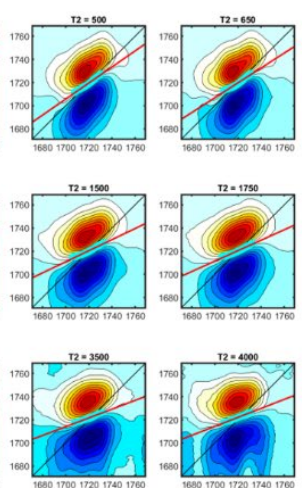

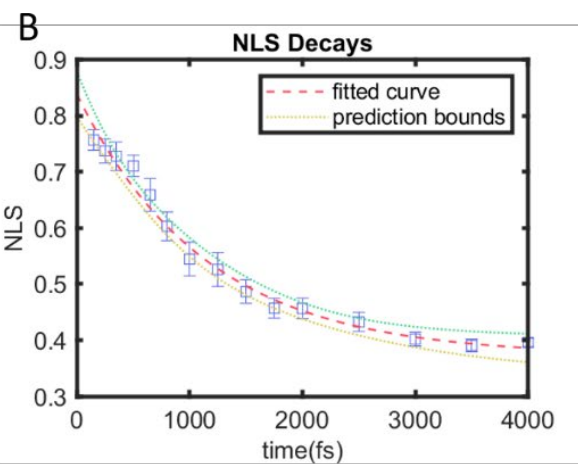

Figure S7. A) 2D IR spectra of the DMPC carbonyl stretch in $200 \mathrm{mg} / \mathrm{mL}$ glucose solutions in $\mathrm{D}_{2} \mathrm{O}$ at selected waiting time delays $\left(\mathrm{t}_{2}\right)$. The horizontal axis represents the 
pump frequency $\left(\omega_{1}\right)$, and the vertical axis is the probe frequency $\left(\omega_{3}\right)$. B) Nodal line slope analysis of the corresponding 2D IR spectra. The red solid line represents a monoexponential fit with an offset to the waiting-time-dependent NLS.

A
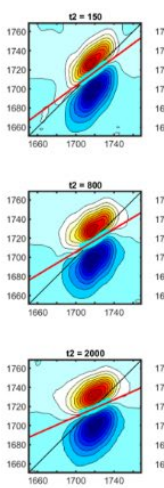
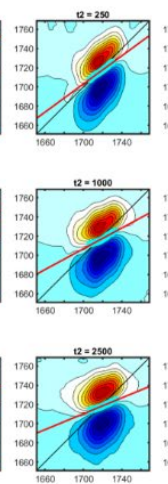
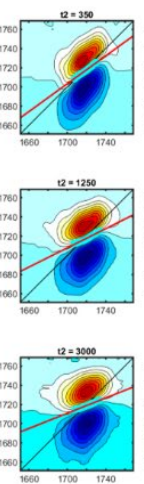
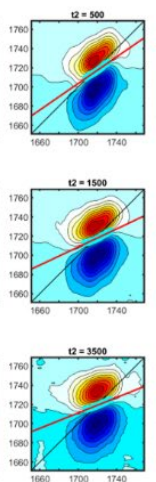
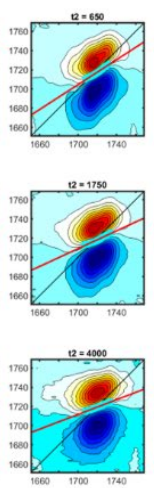

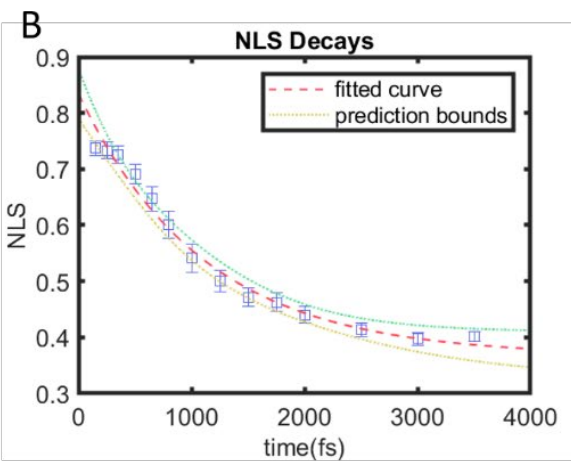

Figure S8. A) 2D IR spectra of the DMPC carbonyl stretch in $300 \mathrm{mg} / \mathrm{mL}$ glucose solutions in $\mathrm{D}_{2} \mathrm{O}$ at selected waiting time delays $\left(\mathrm{t}_{2}\right)$. The horizontal axis represents the pump frequency $\left(\omega_{1}\right)$, and the vertical axis is the probe frequency $\left(\omega_{3}\right)$. B) Nodal line slope analysis of the corresponding 2D IR spectra. The red solid line represents a monoexponential fit with an offset to the waiting-time-dependent NLS.

A
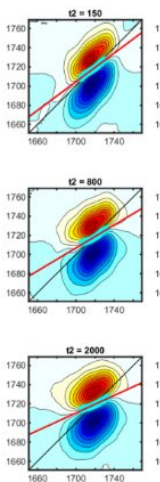
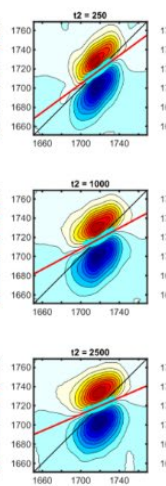
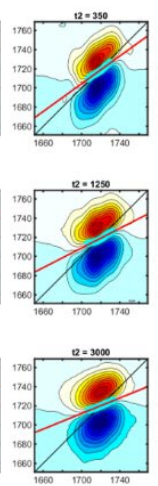
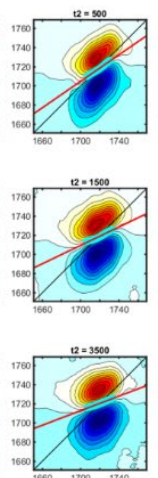


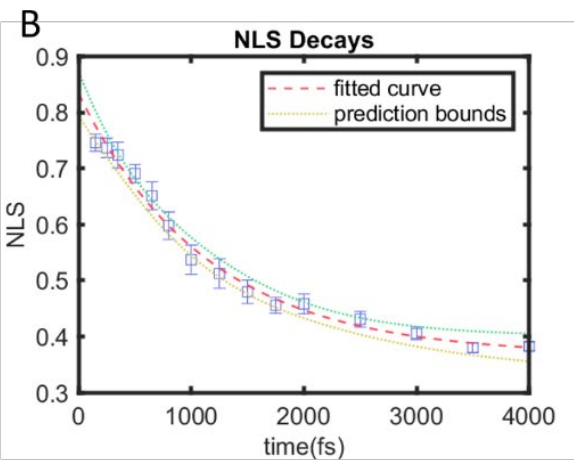

Figure S9. A) 2D IR spectra of the DMPC carbonyl stretch in $400 \mathrm{mg} / \mathrm{mL}$ glucose solutions in $\mathrm{D}_{2} \mathrm{O}$ at selected waiting time delays $\left(\mathrm{t}_{2}\right)$. The horizontal axis represents the pump frequency $\left(\omega_{1}\right)$, and the vertical axis is the probe frequency $\left(\omega_{3}\right)$. B) Nodal line slope 
analysis of the corresponding 2D IR spectra. The red solid line represents a monoexponential fit with an offset to the waiting-time-dependent NLS.

A
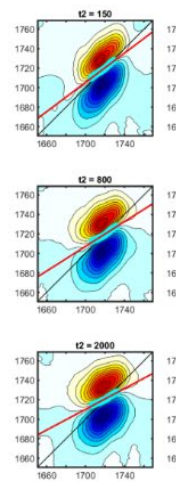
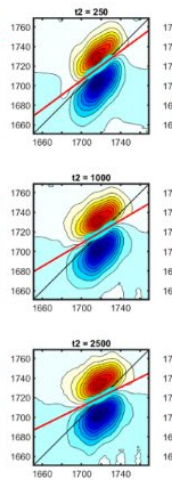
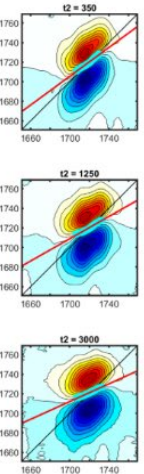
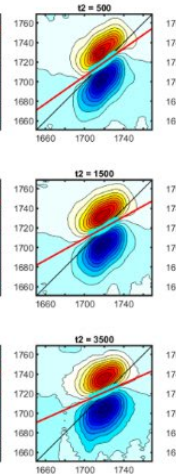
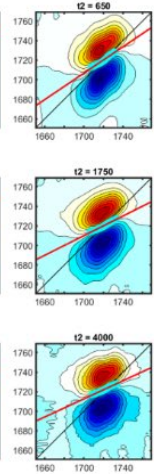

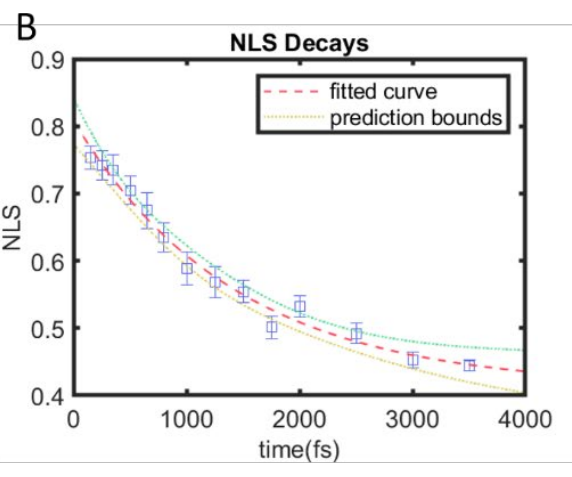

Figure S10. A) 2D IR spectra of the DMPC carbonyl stretch in $500 \mathrm{mg} / \mathrm{mL}$ glucose solutions in $\mathrm{D}_{2} \mathrm{O}$ at selected waiting time delays $\left(\mathrm{t}_{2}\right)$. The horizontal axis represents the pump frequency $\left(\omega_{1}\right)$, and the vertical axis is the probe frequency $\left(\omega_{3}\right)$. B) Nodal line slope analysis of the corresponding 2D IR spectra. The red solid line represents a monoexponential fit with an offset to the waiting-time-dependent NLS.

A
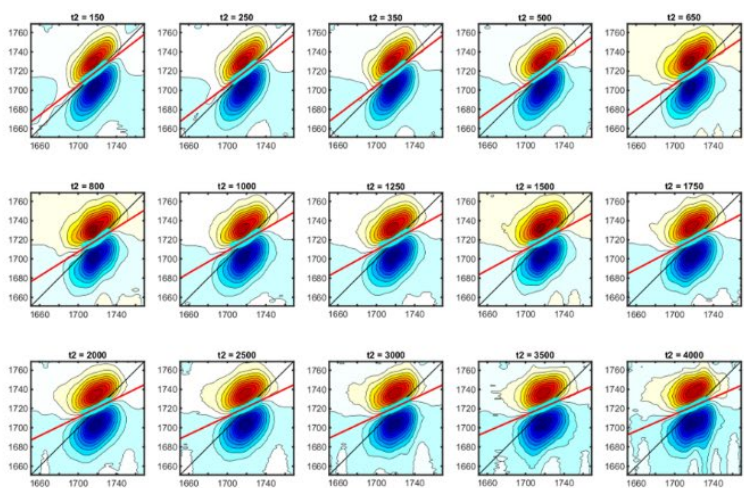
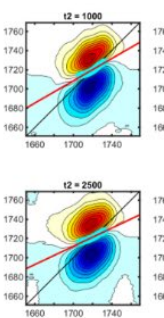
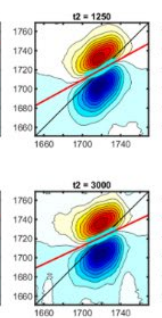
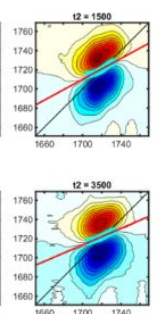
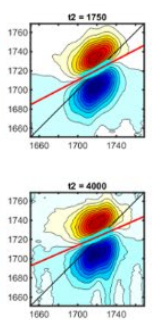

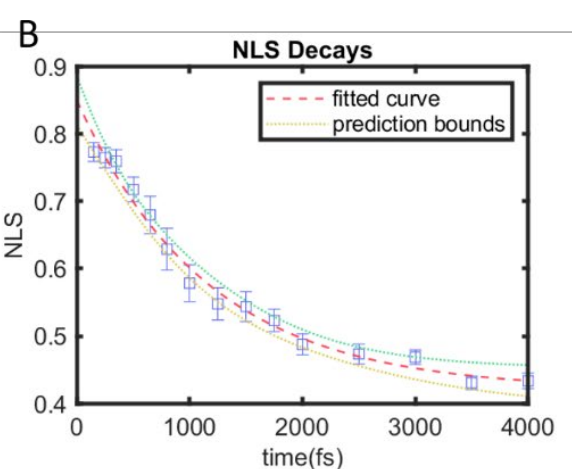

Figure S11. A) 2D IR spectra of the DMPC carbonyl stretch in $600 \mathrm{mg} / \mathrm{mL}$ glucose solutions in $\mathrm{D}_{2} \mathrm{O}$ at selected waiting time delays $\left(\mathrm{t}_{2}\right)$. The horizontal axis represents the pump frequency $\left(\omega_{1}\right)$, and the vertical axis is the probe frequency $\left(\omega_{3}\right)$. B) Nodal line slope analysis of the corresponding 2D IR spectra. The red solid line represents a monoexponential fit with an offset to the waiting-time-dependent NLS. 


\section{S5. Molecular Dynamics Simulations}

\section{DMPC lipid bilayer with glucose}

All-atom MD simulations are carried out using the GROMACS package ${ }^{7}$ employing the CHARMM36 general forcefield. ${ }^{8}$ An initial DMPC lipid bilayer having 100 DMPC lipid molecules was built using the CHARMM membrane builder GUI. ${ }^{9-12}$ Then, simulation boxes composed of the lipid bilayer, water, and glucose molecules were generated using the Packmol program. ${ }^{13}$ To compare with the experiment directly, we made each periodic box which contains the single lipid bilayer, 5000 TIP3P water and 0, $50(100 \% \mathrm{w} / \mathrm{v}), 100$ $(200 \% \mathrm{w} / \mathrm{v}), 150(300 \% \mathrm{w} / \mathrm{v}), 200(400 \% \mathrm{w} / \mathrm{v}), 250(500 \% \mathrm{w} / \mathrm{v})$ and $300(600 \% \mathrm{w} / \mathrm{v})$ glucose molecules, respectively.

In the simulation, the cutoff distance of $12 \AA$ was applied for the Lennard-Jones interaction and the real space part of the Ewald sum, and the particle mesh Ewald (PME) method ${ }^{14}$ was used for long-range electrostatic interactions. We applied the SHAKE algorithm for the TIP3P water with a time step of $1 \mathrm{fs}$.

First, the initial system was energy-minimized for 10000 steps using the steepest-descent energy minimization method. After that, a 20 ns NPT equilibration under a pressure of 1 atom using the Berendsen barostat with a semiisotropic pressure coupling and a temperature of $333 \mathrm{~K}$ was performed using the V-rescale thermostat ${ }^{15}$ with position restraints on the lipid molecules (force constant of $1000 \mathrm{~kJ} \cdot \mathrm{mol}^{-1} \cdot \mathrm{nm}^{-2}$ ). Next, a $5 \mathrm{~ns}$ NVT equilibration with position restraints on the lipid molecules and additional 5 ns NVT equilibration without position restraints were performed at $333 \mathrm{~K}$ using the V-rescale thermostat. Then, a $100 \mathrm{~ns}$ NPT equilibration at $333 \mathrm{~K}$ was carried out using the V-rescale thermostat and the Berendsen barostat. Before we start the production simulation, we performed the additional $20 \mathrm{~ns}$ NVT equilibration to carefully make the thermal equilibrium state of systems after we confirmed a stable area-per-lipid values throughout the production trajectory. Finally, we run the 500 ps NPT production simulation for the carbonyl dynamics of DMPC lipid and the $10 \mathrm{~ns}$ NPT production simulation for the Hbonding network structure analyses between the lipid bilayer, water, and glucose at $333 \mathrm{~K}$ using a Nose-Hoover thermostat and a Parrinello-Raman barostat. Simulation trajectories 
were saved every 20 fs for the 500 ps simulation and every 1 ps for the 10 ns simulation, respectively.

\section{Ethyl Acetate with sugar systems}

For the bulk glucose simulation, we made each periodic box with the same numbers of water and glucose molecules as above. Instead of the lipid bilayer, we inserted 20 ethyl acetate (EtOAc) molecules into each box to see the effect of water and glucose on the carbonyl dynamics, i.e., the carbonyl stretch mode of EtOAc.

First, the system was energy-minimized for 10000 steps using the steepest-descent energy minimization method. After that, a 5 ns NPT equilibration under a pressure of 1 atm using the Berendsen barostat and a temperature of $333 \mathrm{~K}$ using the V-rescale thermostat was performed. Then, a $5 \mathrm{~ns}$ NVT equilibration at $333 \mathrm{~K}$ was performed using the V-rescale thermostat. Finally, we run the 500 ps NPT production simulation for the carbonyl dynamics of EtOAc and the $10 \mathrm{~ns}$ NPT production simulation for the H-bonding network structure analyses between EtOAc, water, and glucose at $333 \mathrm{~K}$ using a Nose-Hoover thermostat and a Parrinello-Raman barostat. Simulation trajectories were saved every 20 fs for the 500 ps simulation and every 1 ps for the $10 \mathrm{~ns}$ simulation, respectively.

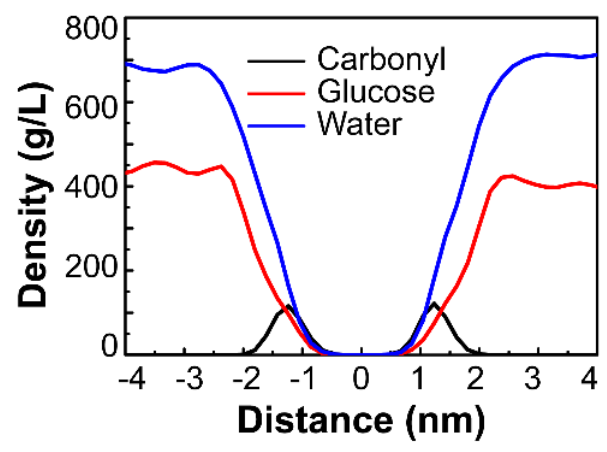

Figure S12. Density profiles along the axis perpendicular to the lipid membrane show that the distribution of carbonyl groups in the DMPC lipids systems locate at the lipid-watersugar interface. 

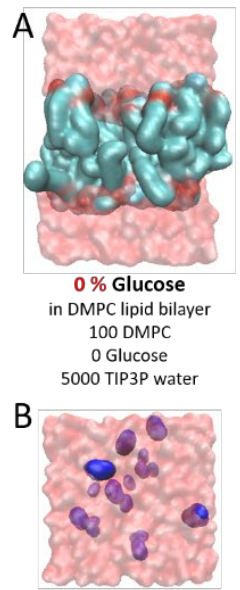

$0 \%$ Glucose

20 EtOAc

5000 TIP3P water
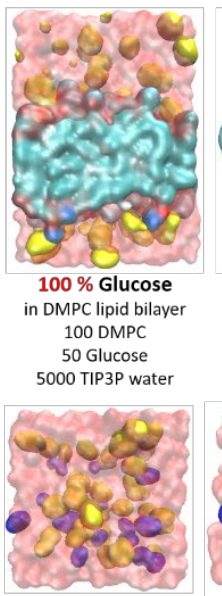

$100 \%$ Glucose

20 EtOAC

5000 TIP3P wate
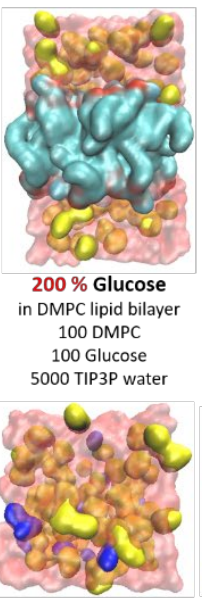

$200 \%$ Glucose

20 EtOAc

100 Glucose
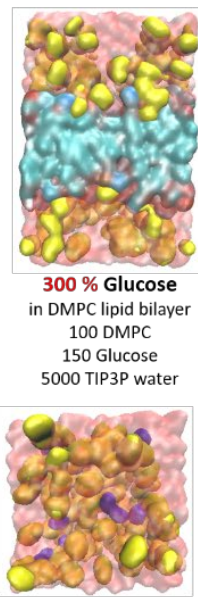

$300 \%$ Glucose

20 EtOAc

5000 TIP3P wate

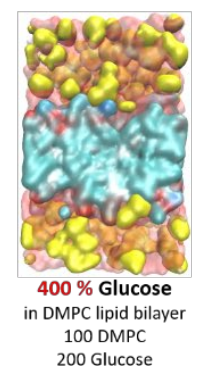

5000 TIP3P water

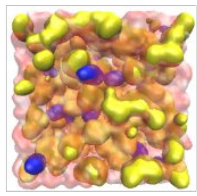

$400 \%$ Glucose

20 EtOAc

5000 TIP3P water
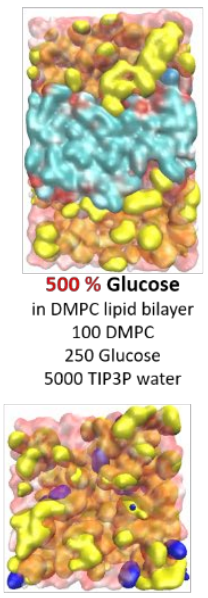

$500 \%$ Glucose

20 EtOAc

5000 TIP3P water
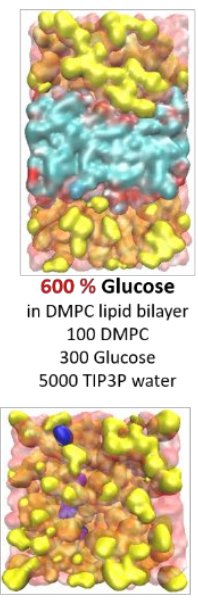

$600 \%$ Glucose

20 EtOAC

5000 TIP3P water

Figure S13. Composition of the simulated systems (description) and equilibrated MD simulation box (image) of A) DMPC Bilayer; B) EtOAc in glucose solution of various concentrations

\section{Calculation of FFCF}
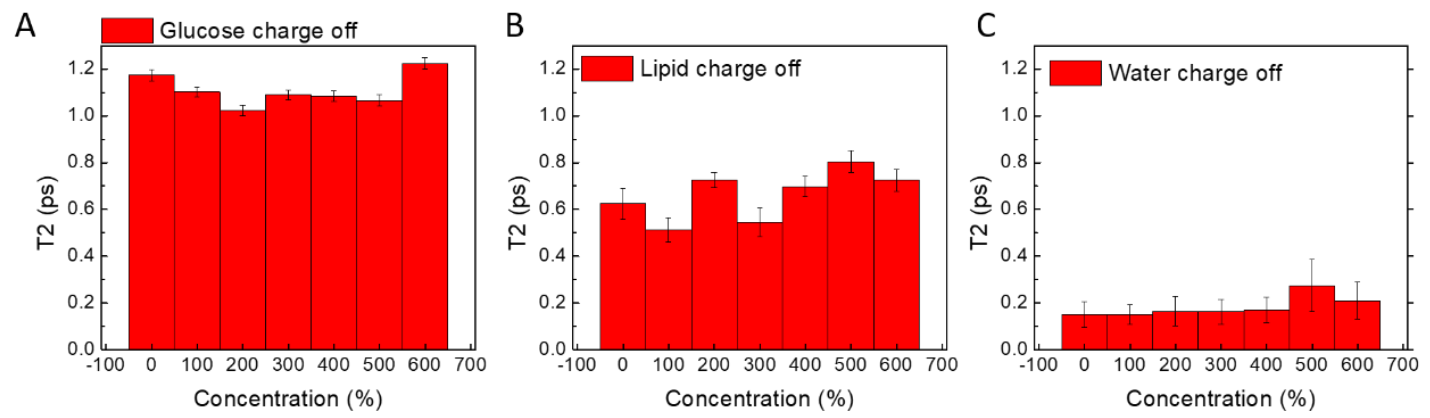

Figure S14. A) Picosecond component of the relaxation time of DMPC carbonyl stretch frequency-frequency correlation function; B-D) Frequency-frequency relaxation of DMPC carbonyl stretch mode with B) glucose C) lipids D) water charges turned off. The figures show dramatic trend and time scale change after the water charge is turned off, meaning that water is the major contribution to the dynamics of carbonyl stretch mode, while glucose influences the trend in a minor way.

Experimental NLS relaxation rates can be compared with the frequency-frequency correlation function (FFCF) from the MD simulation. The FFCF is defined as 


$$
C(t)=\langle\omega(0) \omega(t)\rangle
$$

where $\omega(t)$ is a frequency of the carbonyl stretch mode at time $t$ and the angled brackets indicate an average over the ensemble. The frequency trajectories were obtained using the ester carbonyl frequency map which was developed by our group. ${ }^{16}$ After we get the frequency trajectories and their correlation functions, we fitted FFCFs using a threeexponential decay function. Among three relaxation times, $\mathrm{T} 2$ directly corresponds to the experimental NLS relaxation (Figure 2B).

\section{Calculation of H-bond Properties}

$\mathrm{H}$-bond populations were computed from the production MD trajectories by applying a geometric criterion as implemented in the gmx hbond GROMACS analysis tool. In brief, the donor-acceptor distance cutoff is set to $0.35 \mathrm{~nm}$ and the Donor-H-Acceptor cutoff angle is 30 degrees. These values are determined by radial and angular distribution functions (see for example Figure S17, below)
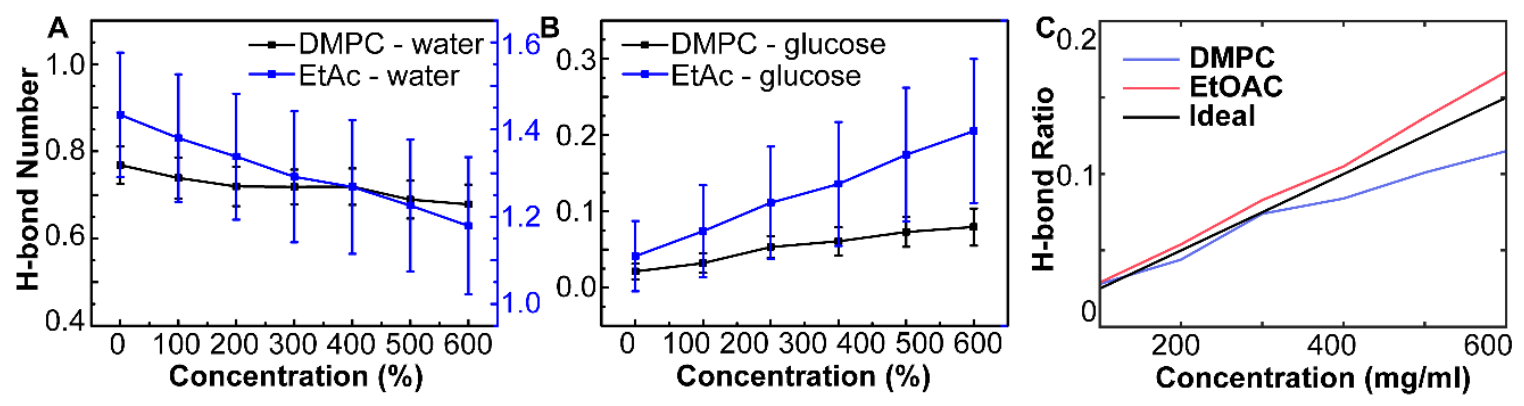

Figure S15. Number of H-bond between A) carbonyl group and water; B) carbonyl group and glucose; C) Ratio of carbonyl-glucose and carbonyl-water H-bonds.

\begin{tabular}{|l|c|c|c|c|c|c|}
\hline Glucose/water & $\mathbf{0 . 0 1}$ & $\mathbf{0 . 0 2}$ & $\mathbf{0 . 0 3}$ & $\mathbf{0 . 0 4}$ & $\mathbf{0 . 0 5}$ & $\mathbf{0 . 0 6}$ \\
\hline Ideal Solution & 0.025 & 0.050 & 0.075 & 0.100 & 0.125 & 0.150 \\
\hline DMPC & 0.028 & 0.044 & 0.074 & 0.084 & 0.101 & 0.115 \\
\hline EtOAC & 0.029 & 0.054 & 0.083 & 0.105 & 0.137 & 0.167 \\
\hline
\end{tabular}


Table S1. Glucose/water molar ratio; calculated lipid-glucose/lipid-water H-bond ratio in ideal solution with no H-bonding preference; MD calculated lipid-glucose/lipid-water Hbond ratio with DMPC carbonyl groups.

The H-bond preference index is obtained by dividing the MD calculated lipidglucose/lipid-water H-bond ratio in DMPC (blue) and EtOAc (red) with the ratio expected in an ideal solution (no preference) in Figure S13C. For example, in the $100 \mathrm{mg} / \mathrm{mL}$ glucose solution, the glucose/water molar ratio is 0.01 . Given that each glucose molecule has five polar hydrogens, while each water has two polar hydrogens, the H-bond donor ratio between glucose and water is 0.25 at this concentration.

\begin{tabular}{|l|c|c|c|c|c|c|}
\hline Molar Ratio & $\mathbf{0 . 0 1}$ & $\mathbf{0 . 0 2}$ & $\mathbf{0 . 0 3}$ & $\mathbf{0 . 0 4}$ & $\mathbf{0 . 0 5}$ & $\mathbf{0 . 0 6}$ \\
\hline DMPC & 1.102 & 0.872 & 0.986 & 0.843 & 0.812 & 0.768 \\
\hline EtOAC & 1.150 & 1.073 & 1.109 & 1.051 & 1.098 & 1.116 \\
\hline
\end{tabular}

Table S2. H-bonding preference index of DMPC and EtOAc; $>1$ value means that carbonyl groups in lipids prefer to bind with glucose; $<1$ indicates preferential $\mathrm{H}$-bonding with glucose.
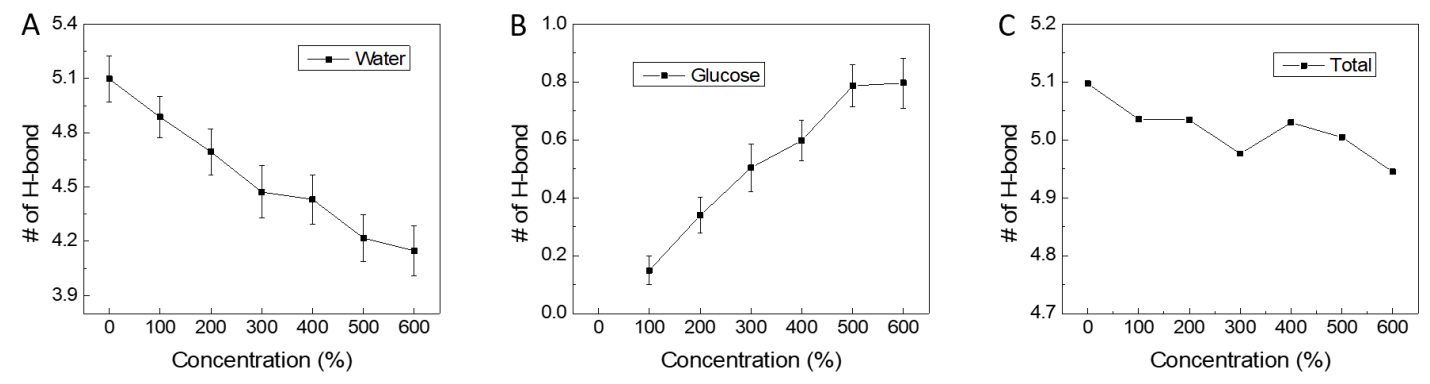

Figure S16. Number of H-bonds between A) DMPC phosphate group and water; B) phosphate group and glucose; C) Total number of H-bonds to the phosphate groups. The $\mathrm{H}$-bond populations show little dependence on glucose concentration indicating that the environment around the phosphate headgroup is also largely preserved across the glucose concentration range. 

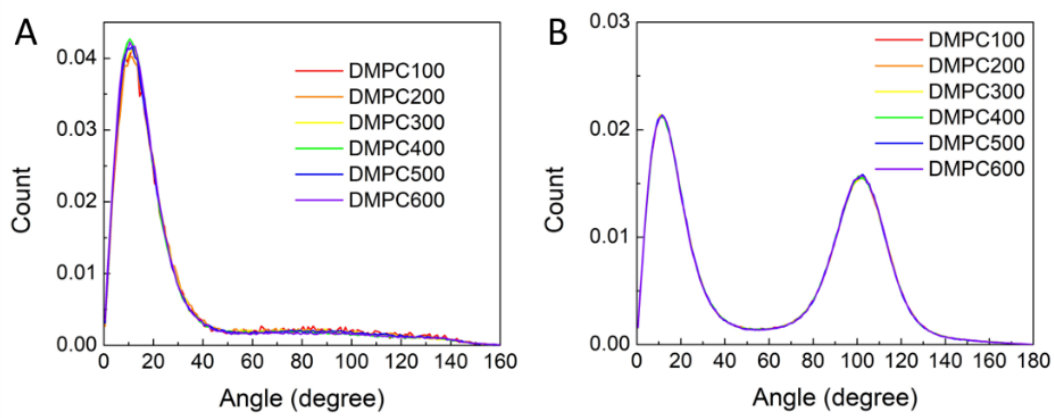

Figure S17. A) Orientational distribution function (ODF) of DMPC carbonyl-associated glucose. B) ODF of DMPC carbonyl-associated water. Here, the angle is defined as the angle between the glucose or water $\mathrm{O}-\mathrm{H}$ vector and vector connecting the carbonyl oxygen (i.e., acceptor) and glucose/water oxygen (i.e., donor). The second peak in (B) represents interactions that lack favorable $\mathrm{H}$-bonding geometries and are therefore not considered $\mathrm{H}$ bond based on the geometric criteria described above.
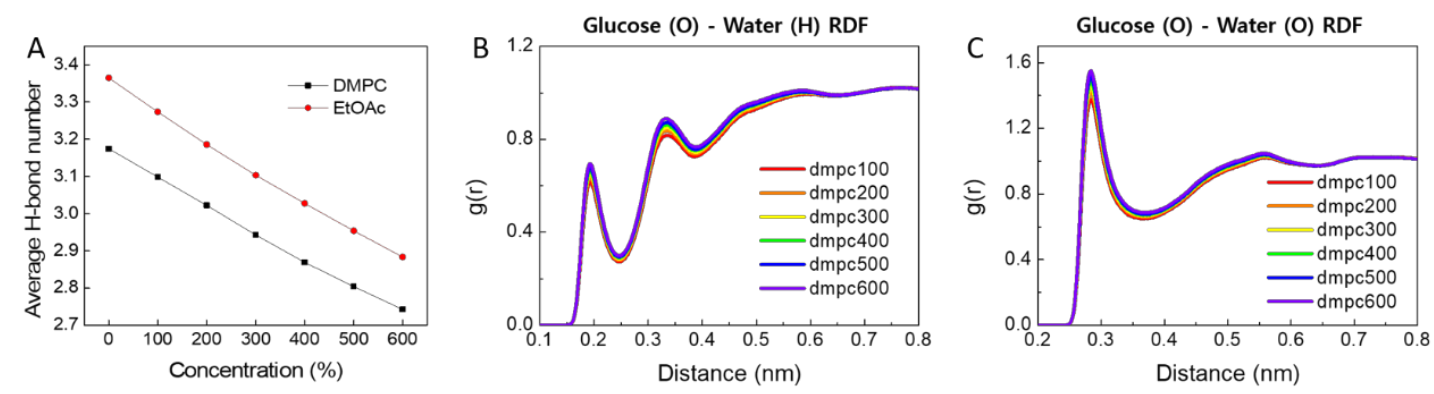

Figure S18. A) Average water-water H-bond number per water molecule as a function of glucose concentration in DMPC lipid bilayer and EtOAc systems. B-C) Radial distribution functions (RDFs) between water $\mathrm{O}$ atom and glucose $\mathrm{O}$ atom $(\mathrm{B})$, and between water $\mathrm{H}$ atom and glucose $\mathrm{O}$ atom $(\mathrm{C})$.

We calculated the average $\mathrm{H}$-bond number per water molecule as a function of glucose concentration in both DMPC and EtOAc systems (Figure S18A). Here, we only considered the H-bond between water molecules. As glucose concentration increases, the average water-water H-bond numbers of both systems monotonically decrease, indicating there is no additional clustering effect on the water H-bond network. Not only the average water- 
water H-bond number, but also RDFs between glucose and water show the weak effect of the glucose concentration change on the interaction between glucose and water (Figure S18B). Even at very high concentrations, peak intensities just slightly increased with increasing glucose concentration.

\section{Calculation of Water Order Parameters}

The ordering of the H-bond network of water is described through the tetrahedral order parameter, $q$. This parameter measures the projection of a water molecule's H-bond geometry onto a perfect tetrahedron. ${ }^{17-18}$ Quantitatively, $q$ is defined as: ${ }^{18-19}$

$$
q=1-\frac{3}{8} \sum_{j=1}^{3} \sum_{k=j+1}^{4}\left(\cos \left(\psi_{j k}\right)+\frac{1}{3}\right)^{2}
$$

where $\psi_{j k}$ represents the angle formed by the central water oxygen atom with each pair of its nearest neighbors (illustration in Figure 2A). In hexagonal ice, all the angles are $109.5^{\circ}$, giving the parameter a value of $q=1$. In an ideal gas, random positions and orientations result in a value of $\langle q\rangle=0 .^{20}$

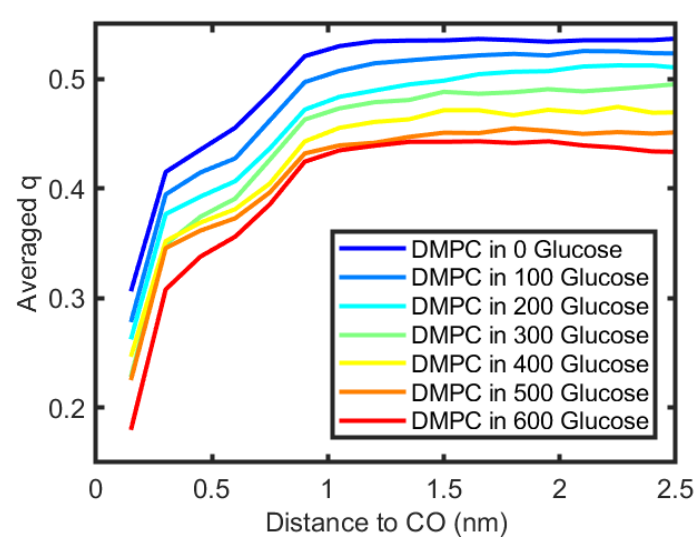

Figure S19. Averaged retrahedral order parameter, $q$, at various distributions at each sugar concentration. The interfacial water is increasingly disturbed at higher sugar concentration resulting in a smaller q value. The extended water network is roughly the size of second to third solvation shell $(0.7 \sim 1 \mathrm{~nm})$ at low sugar concentrations, while the lipid interface has a larger range of impact at higher sugar concentrations. 


\section{Diffusion Coefficients of Water}

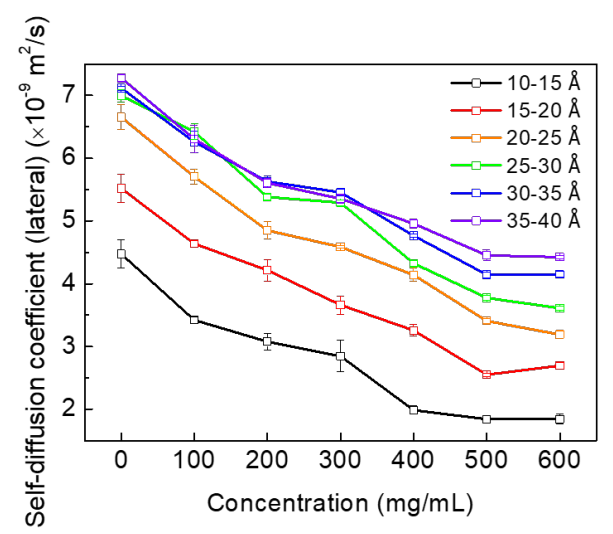

Figure S20. Self-diffusion coefficients of water as a function of glucose concentration. Different-colored lines represent different regions with $5 \AA$ thickness with respect to the distance from the lipid bilayer center.

To see the effect of the glucose concentration change on the water dynamics, we computed a (lateral) self-diffusion coefficient of water located at different regions with respect to the (z-axis) distance from the lipid bilayer center (Figure S20). Here, the diffusion coefficient $(D)$ can be obtained from the following equation (11)

$$
D=\frac{1}{2 d} \lim _{t \rightarrow \infty} \frac{\left\langle|\boldsymbol{r}(t)-\boldsymbol{r}(0)|^{2}\right\rangle}{t}
$$

where $d(d=2$, lateral) is the dimension of the system and $\boldsymbol{r}(\mathrm{t})$ is the position of water. Obviously, water molecules located inside of the lipid bilayer are slower than those located outside of the lipid bilayer. And, they also slow down with increasing glucose concentration. However, in the most of regions, their decaying patterns look similar, suggesting there is no additional effect of glucose concentration change on the water dynamics.

\section{Reference}

1. Valentine, M. L.; Waterland, M. K.; Fathizadeh, A.; Elber, R.; Baiz, C. R. Interfacial Dynamics in Lipid Membranes: The Effects of Headgroup Structures. J Phys Chem B 2021, 125 (5), 1343-1350. 2. Guerin, A. C.; Riley, K.; Rupnik, K.; Kuroda, D. G. Determining the Energetics of the Hydrogen Bond through FTIR: A Hands-On Physical Chemistry Lab Experiment. Journal of Chemical Education 2016, 93 (6), 1124-1129. 
3. Dudis, D. S.; Everhart, J. B.; Branch, T. M.; Hunnicutt, S. S. Hydrogen Bond Energies of Hydrogen Chloride-Carbonyl Complexes. The Journal of Physical Chemistry 1996, 100 (6), 20832088.

4. Edington, S. C.; Flanagan, J. C.; Baiz, C. R. An Empirical IR Frequency Map for Ester C horizontal lineO Stretching Vibrations. J Phys Chem A 2016, 120 (22), 3888-96.

5. Edington, S. C.; Gonzalez, A.; Middendorf, T. R.; Halling, D. B.; Aldrich, R. W.; Baiz, C. R. Coordination to lanthanide ions distorts binding site conformation in calmodulin. Proc Natl Acad Sci U S A 2018, 115 (14), E3126-E3134.

6. Guo, Q.; Pagano, P.; Li, Y. L.; Kohen, A.; Cheatum, C. M. Line shape analysis of twodimensional infrared spectra. J Chem Phys 2015, 142 (21), 212427.

7. Berendsen, H. J. C.; Vanderspoel, D.; Vandrunen, R. Gromacs - a Message-Passing Parallel Molecular-Dynamics Implementation. Comput Phys Commun 1995, 91 (1-3), 43-56.

8. Klauda, J. B.; Venable, R. M.; Freites, J. A.; O'Connor, J. W.; Tobias, D. J.; MondragonRamirez, C.; Vorobyov, I.; MacKerell, A. D., Jr.; Pastor, R. W. Update of the CHARMM all-atom additive force field for lipids: validation on six lipid types. J Phys Chem B 2010, 114 (23), 7830-43.

9. Jo, S.; Kim, T.; Iyer, V. G.; Im, W. Software news and updates - CHARNIM-GUI: A web-based grraphical user interface for CHARMM. J Comput Chem 2008, 29 (11), 1859-1865.

10. Wu, E. L.; Cheng, X.; Jo, S.; Rui, H.; Song, K. C.; Davila-Contreras, E. M.; Qi, Y. F.; Lee, J. M.; Monje-Galvan, V.; Venable, R. M.; Klauda, J. B.; Im, W. CHARMM-GUI Membrane Builder Toward Realistic Biological Membrane Simulations. J Comput Chem 2014, 35 (27), 1997-2004.

11. Jo, S.; Lim, J. B.; Klauda, J. B.; Im, W. CHARMM-GUI Membrane Builder for Mixed Bilayers and Its Application to Yeast Membranes. Biophys J 2009, 97 (1), 50-58.

12. Jo, S.; Kim, T.; Im, W. Automated Builder and Database of Protein/Membrane Complexes for Molecular Dynamics Simulations. Plos One 2007, 2 (9).

13. Martinez, L.; Andrade, R.; Birgin, E. G.; Martinez, J. M. PACKMOL: A Package for Building Initial Configurations for Molecular Dynamics Simulations. J Comput Chem 2009, 30 (13), 21572164.

14. Darden, T.; York, D.; Pedersen, L. Particle Mesh Ewald - an N.Log(N) Method for Ewald Sums in Large Systems. J Chem Phys 1993, 98 (12), 10089-10092.

15. Bussi, G.; Donadio, D.; Parrinello, M. Canonical sampling through velocity rescaling. J Chem Phys 2007, 126 (1).

16. Edington, S. C.; Flanagan, J. C.; Baiz, C. R. An Empirical IR Frequency Map for Ester C=O Stretching Vibrations. J Phys Chem A 2016, 120 (22), 3888-3896.

17. Truskett, T. M.; Torquato, S.; Debenedetti, P. G. Towards a quantification of disorder in materials: distinguishing equilibrium and glassy sphere packings. Phys Rev E Stat Phys Plasmas Fluids Relat Interdiscip Topics 2000, 62 (1 Pt B), 993-1001.

18. Errington, J. R.; Debenedetti, P. G. Relationship between structural order and the anomalies of liquid water. Nature 2001, 409 (6818), 318-21.

19. Chau, P. L.; Hardwick, A. J. A new order parameter for tetrahedral configurations. Molecular Physics 2010, 93 (3), 511-518.

20. Duboue-Dijon, E.; Laage, D. Characterization of the Local Structure in Liquid Water by Various Order Parameters. J Phys Chem B 2015, 119 (26), 8406-18. 